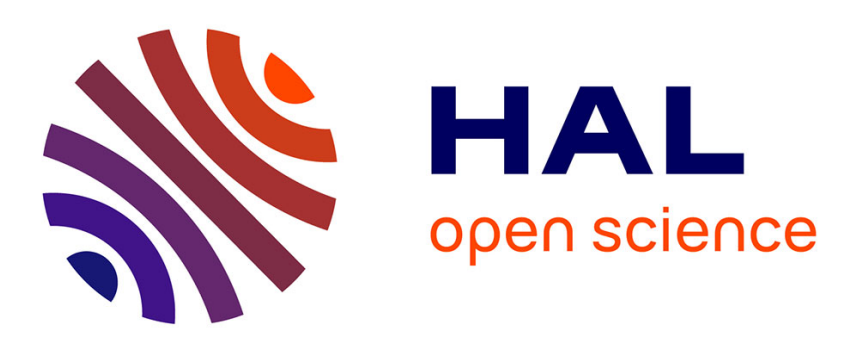

\title{
Magnetworks: How Mobility Impacts the Design of Mobile Ad Hoc Networks
}

Alonso Silva, Eitan Altman, Mérouane Debbah, Giuseppa Alfano

\section{To cite this version:}

Alonso Silva, Eitan Altman, Mérouane Debbah, Giuseppa Alfano. Magnetworks: How Mobility Impacts the Design of Mobile Ad Hoc Networks. IEEE INFOCOM, Mar 2010, San Diego, United States. pp.1 - 9, 10.1109/INFCOM.2010.5461992 . hal-01076473

\section{HAL Id: hal-01076473 \\ https://hal.inria.fr/hal-01076473}

Submitted on 22 Oct 2014

HAL is a multi-disciplinary open access archive for the deposit and dissemination of scientific research documents, whether they are published or not. The documents may come from teaching and research institutions in France or abroad, or from public or private research centers.
L'archive ouverte pluridisciplinaire HAL, est destinée au dépôt et à la diffusion de documents scientifiques de niveau recherche, publiés ou non, émanant des établissements d'enseignement et de recherche français ou étrangers, des laboratoires publics ou privés. 


\title{
Magnetworks: how mobility impacts the design of mobile ad hoc networks
}

\author{
Alonso Silva ${ }^{\dagger}$, Eitan Altman*, \\ Mérouane Debbah, Giuseppa Alfano ${ }^{\dagger}$
}

\begin{abstract}
In this paper we study the optimal placement and optimal number of active relay nodes through the traffic density in mobile sensor ad hoc networks. We consider a setting in which a set of mobile sensor sources is creating data and a set of mobile sensor destinations is receiving that data through multihop wireless paths.
\end{abstract}

We make the assumption that the network is massively dense, i.e., there are so many sources, destinations, and relay nodes, that it is best to describe the network in terms of macroscopic parameters rather than in terms of microscopic parameters.

A simple one-dimensional scenario is used to introduce the problem. We solve the two-dimensional scenario where the mobility of the nodes is deterministic and when it follows the brownian mobility model.

\section{INTRODUCTION}

Various approaches inspired by physics have been proposed to deal with the routing problem in massively dense wireless sensor networks. Starting with the pioneering works of Jacquet [1], [2], and Kalantari and Shayman [3], [4], a number of research groups have worked on this routing problem.

The approach used in [1] and [2] is based on geometrical optics to deal with the case of one source and one destination and a distribution of relay nodes. Approaches based on electrostatics have been studied in [3], [4], [5], [6], [7], [8] (see the survey [9] and references therein) to deal with the case of a distribution of sources and destinations with a density of relay nodes in a static environment.

The development of the theory of routing in massively dense wireless sensor networks has emerged in a complete independent way of the theory developed within the community of road traffic engineers, introduced in 1952 by Wardrop [13] and Beckmann [14], see also [15, pp. 644, footnote 3] for the abundant literature of the early 50's, and which is still an active research area among that community, see [16], [17], [18], [19], [20], and references therein.

Only very recently, new approaches based on road traffic theory have been studied in [10], [11], [12], defining the Wardrop equilibrium and a characterization of the Wardrop equilibrium in this type of setting and including a geometrical characterization of the flow of information for some particular cost functions.

Consider that a spatially distributed set of mobile sources is creating data that must be delivered to a spatially distributed set of mobile destinations. In this context, our objective is to study the optimal traffic distribution and to find the minimum amount of relay nodes needed to transport the data from the sources to the destinations.

The main contribution of this work is to address this problem for a mobile context where we analyze the cases where (i) only sources are mobile and the destinations are static as it would be in the case when the aggregation centers are fixed and the sensor nodes have the capability to move, (ii) the case when both sources and destinations are mobile, and (given that the mathematics involved are similar) (iii) we also analyze the case when the sources are static and the destinations are mobile.

In Section II-A we present a simple one-dimensional scenario to introduce the problem: we analyze the case where the sources and destinations are static and spatially distributed in the one-dimensional line in order to illustrate the behavior of the flow function and the optimal location of the relay nodes on this simple case. We analyze in Section II-B the case when the sources and destinations are static and spatially distributed in the two-dimensional plane. In Section III we analyze the case where the sources and destinations can move with a deterministic velocity and we are able to find the optimal flow of information and to give the optimal spatial density of the relay nodes at each time. Within this section we also give an example with numerical results related to the previous mobility setting. In Section IV we are able to find the optimal spatial density of the relay nodes for the Brownian mobility model. In Section VI we summarize the main results and future perspectives for the continuation of our work. 


\section{THE MODEL}

We first consider the one-dimensional case in order to explain the main concepts involved in our model and how this concepts can be extended to the two-dimensional case in order to obtain the optimal deployment of the relay nodes in a wireless sensor network.

\section{A. Fluid Approximations}

Consider that in the line segment $[0, L]$, which will be the geographical reference of a network, there are wireless sensor nodes.

We consider the continuous node density function $\eta(x)$, measured in nodes $/ \mathrm{m}$, such that the total number of nodes on a segment $\left[\ell_{0}, \ell_{1}\right]$, denoted by $N\left(\ell_{0}, \ell_{1}\right)$, is

$$
N\left(\ell_{0}, \ell_{1}\right)=\int_{\ell_{0}}^{\ell_{1}} \eta(x) d x .
$$

We consider as well the continuous information density function $\rho(x)$, measured in $\mathrm{bps} / \mathrm{m}$, generated by the sensor nodes such that

- At locations $x$ where $\rho(x)>0$ there is a fraction of data created by the sensor sources, such that the rate with which information is created in an infinitesimal line of size $d \varepsilon$, centered at position $x$, is equal to $\rho(x) d \varepsilon$.

- Similarly, at locations $x$ where $\rho(x)<0$ there is a fraction of data received at the sensor destinations such that the rate with which information is received by an infinitesimal line of size $d \varepsilon$, centered at position $x$, is equal to $-\rho(x) d \varepsilon$.

We assume that the total rate at which sensor destinations have to receive data is the same as the total rate which the data is created at the sensor sources, i.e.,

$$
\int_{0}^{L} \rho(x) d x=0 .
$$

Notice that if we have an estimation of the proportion of packet loss in the network, we can weight our function $\rho$ in order to adapt it to equation (1).

Consider the continuously differentiable traffic flow function $T(x)$, measured in bps, such that its direction (positive or negative) coincides with the direction of the flow of information at point $x$ and $|T(x)|$ is the rate at which information propagates at position $x$, i.e., $|T(x)|$ gives the total amount of traffic that is passing through the position $x$.
Next we present the flow conservation condition. For information to be conserved over a segment $\left[\ell_{0}, \ell_{1}\right]$, it is necessary that the rate with which information is created over the segment is equal to the rate with which information is leaving the line segment, i.e.,

$$
T\left(\ell_{1}\right)-T\left(\ell_{0}\right)=\int_{\ell_{0}}^{\ell_{1}} \rho(x) d x .
$$

The integral on the right hand side is equal to the quantity of information generated (if it's positive) or demanded (if it's negative) by the fraction of sensor nodes over the line segment $\left[\ell_{0}, \ell_{1}\right]$. The expression $T\left(\ell_{1}\right)-T\left(\ell_{0}\right)$, measured in $\mathrm{bps} / \mathrm{m}$, is equal at the rate with which information is leaving (if it's positive) or entering (if it's negative) the segment $\left[\ell_{0}, \ell_{1}\right]$. This holding for any line segment, it follows that necessarily,

$$
\frac{d T(x)}{d x}=\rho(x) \text {. }
$$

The problem considered is to minimize the number of nodes $N(0, L)$ in the line segment $[0, L]$, needed to support the information created by the sensor sources and received by the sensor destinations subject to the flow conservation condition given by (2) and imposing that there is no flow of information leaving the network, i.e., $T(0)=0$ and $T(L)=0$. Thus the system of equations that model our problem in the one-dimensional case is given by the system of equations:

$$
\begin{gathered}
\operatorname{Min} N(0, L)=\int_{0}^{L} \eta(x) d x, \\
\text { subject to } \frac{d T(x)}{d x}=\rho(x) \quad \operatorname{in}(0, L), \\
T(0)=0 \quad \text { and } T(L)=0 .
\end{gathered}
$$

Notice that in the one-dimensional case, there is no minimization problem because only by using the constraints (4) and (5), we obtain only one solution. As we will see, this will not be the case for the two-dimensional case.

We suppose that the proportion of sensor nodes $\eta(x)$ in an area of infinitesimal size $d \varepsilon$, centered at location $x$, needed as relay nodes, will be proportional to the traffic flow of information that is passing through that region, i.e., $\eta(x) d \varepsilon=|T(x)|^{\alpha} d \varepsilon$ where $\alpha>$ 0 is a fixed number called the relay-traffic constant. Then the optimal placement of the relay nodes in the network will be given by $\eta^{*}(x)=\left|T^{*}(x)\right|^{\alpha}$, where the traffic flow function $T^{*}(x)$ is the optimal traffic flow function, given by the solution of the previous system of equations. Furthermore, the optimal total number of relay 


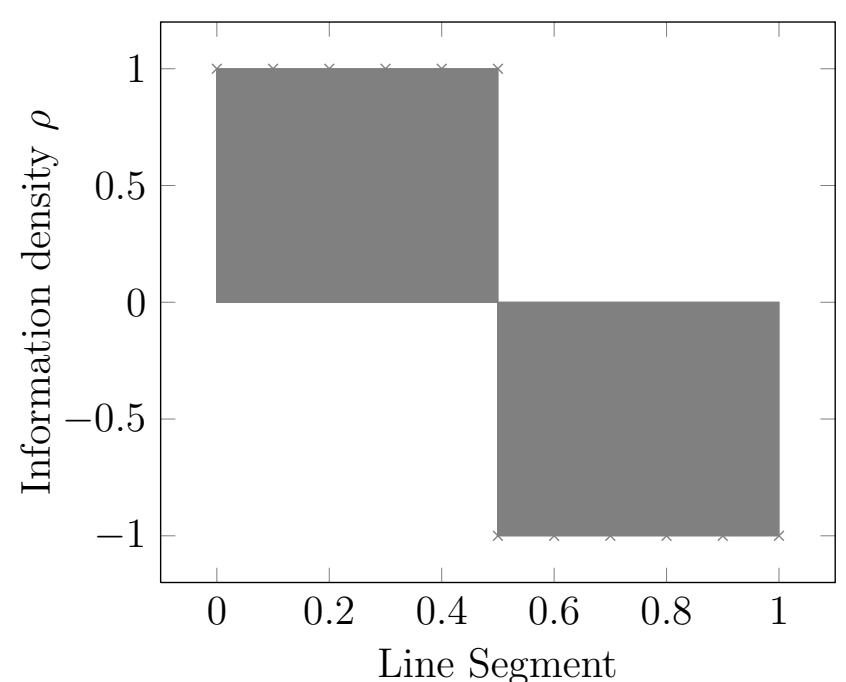

nodes $N^{*}(0, L)$ needed to support the optimal traffic flow function $T^{*}(x)$ in the network will be $N^{*}\left(\ell_{0}, \ell_{1}\right)=$ $\int_{\ell_{0}}^{\ell_{1}} \eta(x) d x=\int_{\ell_{0}}^{\ell_{1}}|T(x)|^{\alpha} d x$. Let us see an example to illustrate the previous framework.

Example 1. Suppose that we can divide the line segment $[0, L]$ in two parts: (i) in the first part $[0, L / 2]$ there will be a uniform information density function generated by the sensor sources, given by $\rho(x)=1$ $\mathrm{bps} / \mathrm{m}$ and (ii) in the second half $[L / 2, L]$ there will be a uniform information density function received at the sensor destinations given by $\rho(x)=-1 \mathrm{bps} / \mathrm{m}$ (See Figure 1). From (4) and (5) we obtain that the optimal traffic flow function will be given by $T^{*}(x)=x \mathrm{bps} / \mathrm{m}$ for all $x \in[0, L / 2]$ and $T^{*}(x)=L-x \mathrm{bps} / \mathrm{m}$ for all $x \in[L / 2, L]$ with positive direction (See Figure 2). If we assume that the relay-traffic constant $\alpha=2$, then the optimal placement of the relay nodes needed to relay the information from the sources to the destinations on the network will be given by $\eta^{*}(x)=x^{2}$ for all $x \in[0, L / 2]$ and $T^{*}(x)=(L-x)^{2} \mathrm{bps} / \mathrm{m}$ for all $x \in[L / 2, L]$.

The optimal total number of relay nodes $N^{*}(0, L)$ needed to support the optimal traffic $T^{*}(x)$ will be given by $N(L)=\int_{0}^{L / 2} x^{2} d x+\int_{L / 2}^{L}(L-x)^{2} d x=L^{3} / 12$.

\section{B. The two-dimensional case}

Consider in a grid area network $D$ in the two dimensional plane ${ }^{1} X \times Y$, the continuous information density function $\rho(\mathbf{x})$, measured in $\mathrm{bps} / \mathrm{m}^{2}$, such that at locations $\mathbf{x}$ where $\rho(\mathbf{x})>0$, there is a distributed

\footnotetext{
${ }^{1}$ We will denote with bold fonts the vectors and $\mathbf{x}=(x, y)$ will denote a location in the two dimensional space $X \times Y$.
}
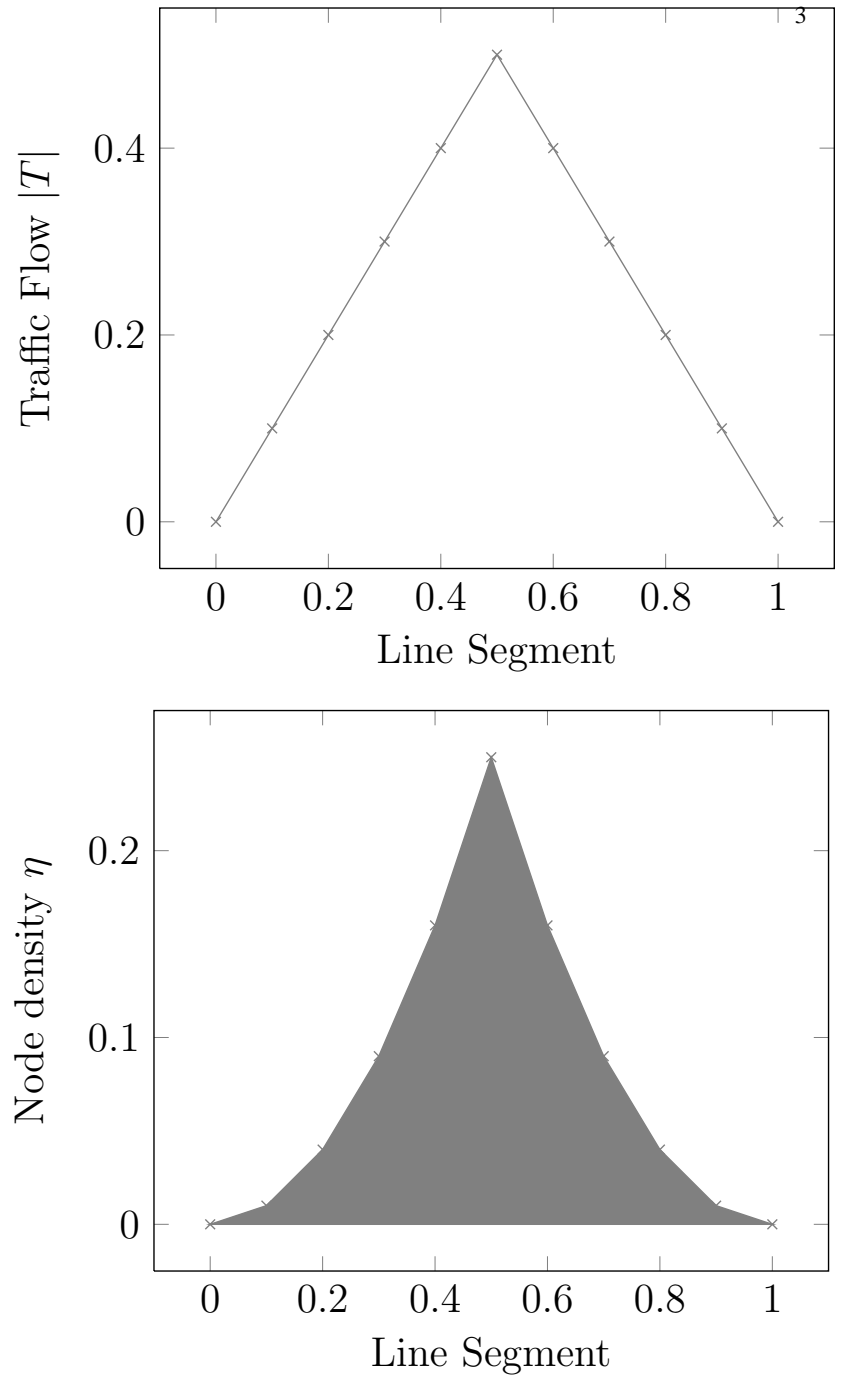

data source created by sensor sources, such that the rate with which information is created in an infinitesimal area of size $d A_{\varepsilon}$, centered at location $\mathbf{x}$, is $\rho(\mathbf{x}) d A_{\varepsilon}$. Similarly, at locations $\mathbf{x}$ where $\rho(\mathbf{x})<0$, there is a distributed data sink received at sensor destinations, such that the rate with which information can be treated by an infinitesimal area of size $d A_{\varepsilon}$, centered at location $\mathbf{x}$, is equal to $-\rho(\mathbf{x}) d A_{\varepsilon}$.

The total rate at which sensor destinations must process data is the same as the total rate which the data is created at the sensor sources, i.e.,

$$
\int_{X \times Y} \rho(\mathbf{x}) d \mathbf{x}=0 .
$$

Consider the continuous node density function $\eta(\mathbf{x})$, measured in nodes $/ \mathrm{m}^{2}$, defined so that the number of 
relay nodes in an area of infinitesimal size $d A_{\varepsilon}$, centered at $\mathbf{x}$, is equal to $\eta(\mathbf{x}) d A_{\varepsilon}$.

The total number of nodes on a region $A$, denoted by $N(A)$, is then given by

$$
N(A)=\int_{A} \eta(\mathbf{x}) d \mathbf{x} .
$$

Consider the continuous traffic flow function $\mathbf{T}(\mathbf{x})$, measured in $\mathrm{bps} / \mathrm{m}$, such that its direction coincides with the direction of the flow of information at point $\mathrm{x}$, and ${ }^{2}$ $\|\mathbf{T}(\mathbf{x})\|$ is the rate with which information rate crosses a linear segment perpendicular to $\mathbf{T}(\mathbf{x})$ centered on $\mathbf{x}$, i.e., $\|\mathbf{T}(\mathbf{x})\| \varepsilon$ gives the total amount of traffic crossing a linear segment of infinitesimal length $\varepsilon$, centered at location $\mathbf{x}$, and placed vertically to $\mathbf{T}(\mathbf{x})$.

Next we present the flow conservation condition (see e.g. [6], [16], for more details about this type of condition). For information to be conserved over a domain $D$ of arbitrary shape on the $X \times Y$ plane, with smooth boundary $\partial D$, it is necessary that the rate with which information is created in the area is equal to the rate with which information is leaving the area, i.e.,

$$
\int_{D} \rho(\mathbf{x}) d D=\oint_{\partial D}[\mathbf{T} \cdot \mathbf{n}(\mathbf{x})] d \ell
$$

The integral on the left is the surface integral of $\rho(\mathbf{x})$ over the domain $D$. The integral on the right is the path integral of the inner product $\mathbf{T} \cdot \mathbf{n}$ over the boundary $\partial D$. The vector $\mathbf{n}(\mathbf{x})$ is the unit normal vector to $\partial D$ at the boundary point $\mathbf{x} \in \partial D$ and pointing outwards. Then the function $\mathbf{T} \cdot \mathbf{n}(\mathbf{x})$, measured in $\mathrm{bps} / \mathrm{m}$, is equal to the rate with which information is leaving the interface $D$ through a unit length line segment at the boundary point $\mathbf{x}$.

This holding for any (smooth) domain $D$, it follows that necessarily

$$
\nabla \cdot \mathbf{T}(\mathbf{x}):=\frac{\partial T_{1}(\mathbf{x})}{\partial x_{1}}+\frac{\partial T_{2}(\mathbf{x})}{\partial x_{2}}=\rho(\mathbf{x})
$$

where " $\nabla \cdot "$ is the divergence operator.

Thus the problem considered is to minimize the quantity of nodes $N(D)$ in the grid area network $D$ needed to support the information created by the distribution of sources subject to the flow conservation condition, i.e.,

\footnotetext{
${ }^{2}$ The norm $\|\cdot\|$ is the Euclidean norm, i.e., for a vector $\mathbf{x}=(x, y)$, its norm will be $\|\mathbf{x}\|=\sqrt{x^{2}+y^{2}}$.
}

our problem is given by the system of equations:

$$
\begin{gathered}
\operatorname{Min} N(D) \\
\text { subject to } \nabla \cdot \mathbf{T}=\rho(\mathbf{x}) .
\end{gathered}
$$

Toumpis and Tassiulas in [5] focus on a particular physical layer model characterized by the following assumption:

Assumption : A location $\mathbf{x}$ where the node density is $\eta(\mathbf{x})$ can support any traffic flow vector with a magnitude less or equal to a bound $\|\mathbf{T}(\mathbf{x})\|_{\max }$ which is proportional to the square root of the density, i.e. $\|\mathbf{T}(\mathbf{x})\| \leq\|\mathbf{T}(\mathbf{x})\|_{\max }=K \sqrt{d(\mathbf{x})}$.

The validity of Assumption 1 depends on the physical layer and the medium access control protocol used by the network. Although it is not generally true, it holds in many different settings of interest. For example, in [5] Toumpis and Tassiulas give an example of network where $m^{2}$ nodes are placed in a perfect square grid of $m \times m$ nodes and each node can listen to transmissions from its four nearest neighbors. They give a simple time division routine so that the network of $\mathrm{m}^{2}$ nodes can support a traffic on the order of $m$. As another example, in [22] it was shown that the traffic that can be supported in the above network, if nodes access the channel by use of slotted Aloha instead of time division, is $\mathbf{T}_{\text {local }}=K \times W \times m$, where nodes transmit data with a fixed global rate of $W$ bps, $K$ is a constant-smaller than 1/3-that captures the efficiency of Aloha. Finally, in [21] it was shown that a network of $n$ randomly placed nodes can support an aggregate traffic on the order of $\sqrt{n / \log n}$ under a more realistic interference model that accounts for interference coming from arbitrarily distant nodes. The logarithm in the denominator appears due to the proving methodology of [21], and it has been shown [23] that it can be dispensed off, by use of percolation theory in the proofs.

Tassiulas and Toumpis prove in [5] that among all traffic flow functions that satisfy $\nabla \cdot \mathbf{T}=\rho$, the one that minimizes the number of nodes needed to support the network, must be irrotational, i.e.,

$$
\nabla \times \mathbf{T}=0
$$

where " $\nabla \times$ " is the curl operator.

\section{MODEL IN MOTION}

In our work we do a parallel to Electromagnetism by considering moving sensor nodes or moving distribution of sensor nodes. In that sense we first define some terms directly related to Electromagnetism. In our model, 
the information density function $\rho$, the traffic flow function $\mathbf{T}$, and the node density function $\eta$, defined previously may depend on time, i.e., $\rho=\rho(\mathbf{x}, t), \mathbf{T}=$ $\mathbf{T}(\mathbf{x}, t)$, and $\eta=\eta(\mathbf{x}, t)$. We will consider our problem within a window of time $t \in\left[t_{i}, t_{f}\right]$ where $t_{i}$ is the initial time and $t_{f}$ is the final time.

We define the continuous node current $\mathbf{J}$ as the density of sensor nodes $\rho(\mathbf{x}, t)$ in the position $\mathbf{x}$ multiplied by the nodes average drift velocity $\mathbf{v}(\mathbf{x}, t)$, i.e.,

$$
\mathbf{J}=\rho(\mathbf{x}, t) \mathbf{v}(\mathbf{x}, t) .
$$

The rate at which nodes leaves an area (or volume) $V$, bounded by a curve (or surface) $S=\partial V$, will be given by

$$
\oint_{S} \mathbf{J} \cdot d S
$$

Since the information density function is conserved in the plane this integral must be equal to

$$
\oint_{S} \mathbf{J} \cdot d S=-\frac{d}{d t} \oint_{S} \rho \cdot \mathbf{n} d S=-\int_{V} \frac{\partial \rho}{\partial t} d V .
$$

From the divergence theorem and imposing the equality between the equations (10) and (11), we obtain the equivalent to Kirchhoff's current law:

$$
\nabla \cdot \mathbf{J}+\frac{\partial \rho}{\partial t}=0
$$

Notice as well that

$$
\nabla \cdot \mathbf{J}=\nabla \cdot(\rho \mathbf{v})=\mathbf{v} \cdot \nabla \rho+\rho \nabla \cdot \mathbf{v} .
$$

We assume that we know the initial distribution of the sensor sources and the sensor destinations at time 0 denoted by $\rho_{0}$. Thus we obtain the following system of equations:

$$
\text { (TE) }\left\{\begin{aligned}
\frac{\partial \rho}{\partial t}+\mathbf{v} \cdot \nabla \rho+\rho \nabla \cdot \mathbf{v}=0 & \text { in } D \times(0, T) \\
\rho(0)=\rho_{0} & \text { on } D \times\{0\} .
\end{aligned}\right.
$$

The previous system of equations is known in the partial differential equations literature as the linear transport equation with initial condition for which there exists a solution (see Proposition II.1 of [24]).

Notice that given the initial distribution of the sources and destinations and the velocity of the distribution, we are able to compute the evolution of the distribution of sensor sources and sensor destinations on time $t \in[0, T)$. The velocity of sensor sources and sensor destinations may be estimated by having some previous knowledge on the behavior of these sources and destinations in our network.
We want to minimize the number of relay nodes $N(D)^{5}$ in the grid area network $D$ needed to support the information created by the distribution of sensor sources and received by the distribution of sensor destinations subject to the flow conservation condition, and knowing that the distribution of mobile sensor nodes and mobile sensor destinations is the solution to the system of equations (TE). Thus our problem reads for all $t \in[0, T]$

$$
\begin{array}{cl}
\operatorname{Min} N(D, t) & =\int_{D} \eta(\mathbf{x}, t) d \mathbf{x}=\int_{D}|\mathbf{T}(\mathbf{x}, t)|^{2} d \mathbf{x} \\
\text { subject to } \quad & \nabla \cdot \mathbf{T}(\mathbf{x}, t)=\rho(\mathbf{x}, t) \text { in } D, \\
& \mathbf{T} \cdot \mathbf{n}=0 \text { on } \partial D .
\end{array}
$$

where $\rho(\mathbf{x}, t)$ is the solution to the problem (TE).

We recall that Tassiulas and Toumpis in [5] proved that among all traffic flow functions that satisfy equation (13a), the one that minimizes the number of nodes needed to support the network, must satisfy

$$
\nabla \times \mathbf{T}=0 .
$$

Using Helmholtz's theorem (also known as fundamental theorem of vector calculus) to last equation (14) we obtain that there exists a scalar potential function $\varphi$ such that

$$
-\nabla \varphi=\mathbf{T}
$$

Replacing this function into the conservation equation (13a) we obtain that

$$
-\Delta \varphi=\rho
$$

where " $\Delta$ " is the Laplacian operator and this holds for all $t \in[0, T)$.

We impose that no information is leaving the considered domain $D$, in equation (13b) and from equation (15) this condition translates into $\nabla \varphi \cdot \mathbf{n}=0$

From equation (16) and last condition we obtain the following system

$$
\text { (LE) }\left\{\begin{aligned}
-\Delta \varphi & =\rho & & \text { in } D \\
\nabla \varphi \cdot \mathbf{n} & =0 & & \text { on } \partial D .
\end{aligned}\right.
$$

which is the Laplace equation with Neumann boundary conditions.

If the function $f$ is square integrable then the Laplace equation with Neumann boundary conditions has a unique solution in $H^{1}(D) / \mathbb{R}$.

In summary in order to solve our problem given by the equations (12), (13a), (13b), and (TE) we need to

1) Solve the system of equations (TE), 
2) Put the solution as input into the system of equations (LE),

3) Solve the system of equations (LE).

Even though it looks complicated system we give an example where we can get explicit solutions.

Example 2. Due to presentation effects we consider the one dimensional case during $T=2$ hours.

We consider an initial distribution of sensor sources $\rho_{0}^{+}$ and an initial distribution of sensor destinations $\rho_{0}^{-}$on the positive real line $[0,+\infty)$, and we scale them to be probability distributions so it represents in each location the proportion of sensor sources or the proportion of sensor destinations respectively:

$$
\rho_{0}^{+}=k_{1} e^{-(x-3)^{2}} \quad \text { and } \quad \rho_{0}^{-}=-k_{2} e^{-(x-10)^{2}} .
$$

where $k_{1}$ and $k_{2}$ are normalization factors given by $k_{1}=\frac{2}{\sqrt{\pi} \operatorname{erfc}(-3)}, \quad k_{2}=\frac{2}{\sqrt{\pi} \operatorname{erfc}(-10)}$, where $\operatorname{erfc}(\mathrm{x})$ is the complementary error function defined as $\operatorname{erfc}(\mathrm{x})=\frac{2}{\sqrt{\pi}} \int_{x}^{+\infty} e^{-s^{2}} d s$.

We consider that the nodes average drift velocity is given by $v(x, t)=x$. We can think of a highway where the cars are equipped with sensors and while they are advancing on the highway they can go faster and faster.

1. We first need to solve the transportation equation system (TE), that in our example reads

$$
\left\{\begin{array}{cl}
\frac{\partial \rho}{\partial t}+\frac{\partial(x \rho)}{\partial x}=0 & \text { on } \mathbb{R}_{+} \times[0, T) \\
\rho(0)=\rho_{0}^{+}+\rho_{0}^{-} & \text {on } \mathbb{R}_{+}
\end{array}\right.
$$

Using the method of characteristics we obtain that the information density function over time is given by $\rho(x, t)=\rho^{+}(x, t)+\rho^{-}(x, t)$ where

$$
\left\{\begin{array}{l}
\rho^{+}(x, t)=k_{1} e^{-\left(x e^{-t}-3\right)^{2}-t} \\
\rho^{-}(x, t)=-k_{2} e^{-\left(x e^{-t}-10\right)^{2}-t}
\end{array}\right.
$$

The solution combining the sources and destinations information density function over time are showed in Fig. 1.

2. We put the solution as input into the system of equations: from the conservation equation we obtain

$$
\frac{\partial T(x, t)}{\partial x}=\frac{\partial T^{+}(x, t)}{\partial x}+\frac{\partial T^{-}(x, t)}{\partial x}
$$

where $\frac{\partial T^{+}(x, t)}{\partial x}=\rho^{+}$and $\quad \frac{\partial T^{-}(x, t)}{\partial x}=-\rho^{-}$.

with initial condition that the flow is zero at the boundary point zero, i.e. $T(0, t)=0$.

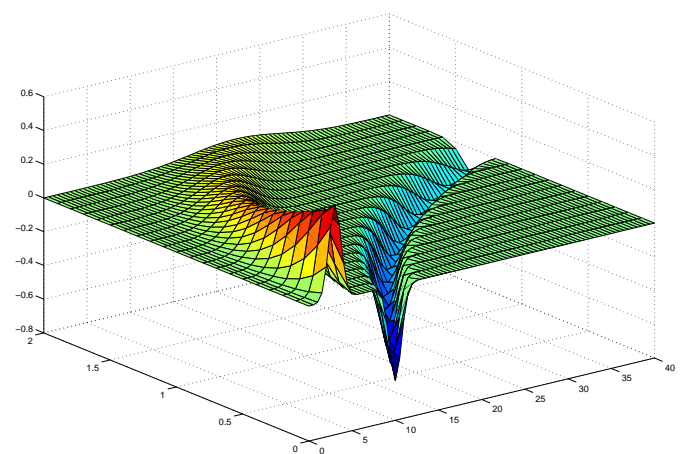

Fig. 1. Distribution of the sources and destinations in the same line

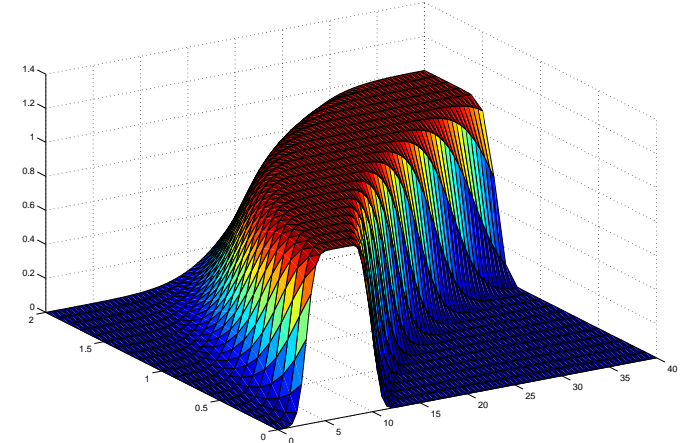

Fig. 2. Optimal traffic flow

3. We solve the Laplacian system of equations: Then the optimal traffic flow function is given by $T^{*}(x, t)=T^{+}(x, t)+T^{-}(x, t)$ where

$$
\left\{\begin{array}{l}
T^{+}(x, t)=\int_{0}^{x} k_{1} e^{-\left(x e^{-t}-3\right)^{2}-t} d x \\
T^{-}(x, t)=-\int_{0}^{x} k_{2} e^{-\left(x e^{-t}-10\right)^{2}-t} d x .
\end{array}\right.
$$

Thus the minimal number of active relay nodes needed to support the optimal flow at every time $t$ will be given by

$$
N^{*}(t)=\int_{0}^{+\infty}\left|T^{*}(x, t)\right|^{2} d x
$$

which can be solved numerically.

In the next section we present another type of mobility model where we consider the randomness in the mobility of the users. 


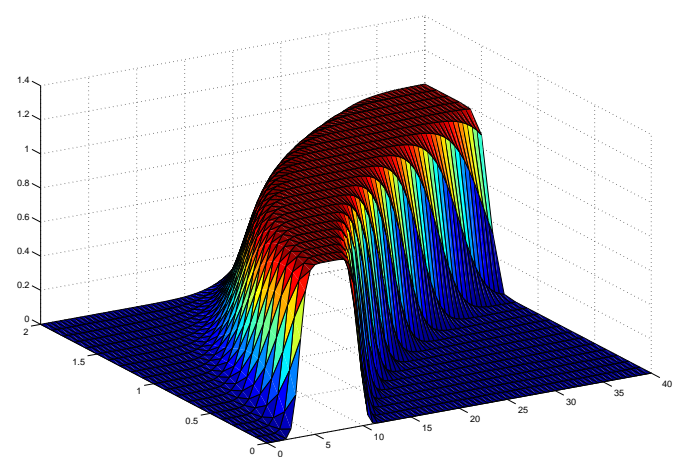

Fig. 3. Optimal relay node distribution

\section{BRownian Mobility Model}

One of the most used mobility models used in networks is the Random Walk Mobility Model also known as the Brownian Mobility Model (see the survey [26] and the references therein).

If we have previous knowledge about the velocity drift of the distribution of information created at the sources (denoted $\rho^{+}$) and/or the distribution of information received at the destinations (denoted $\rho^{-}$), and we assume the Brownian mobility model, then the distribution of sources and/or the distribution of the destinations evolves according to the stochastic differential equation

$$
d \rho^{+}(t)=\mathbf{v}^{+}(\mathbf{x}, t) d t+\sigma_{+}(\mathbf{x}, t) d W^{+}(t)
$$

and/or $\quad d \rho^{-}(t)=\mathbf{v}^{-}(\mathbf{x}, t) d t+\sigma_{-}(\mathbf{x}, t) d W^{-}(t)$.

where $W^{+}(t)$ and $W^{-}(t)$ are two independent brownian motion with values in $X \times Y$ and $\sigma_{+}:=\sigma_{+}(\mathbf{x}, t)$, $\sigma_{-}:=\sigma_{-}(\mathbf{x}, t)$ are parameters of the model.

Assume as in the previous case that we know the initial distribution of the information created at the sources. Then by using Itô's lemma, $\rho^{+}$evolves in time by the Kolmogorov Forward Equation

$\frac{\partial}{\partial s} p(\mathbf{x}, s)=-\frac{\partial}{\partial x}\left[\mathbf{v}^{+}(\mathbf{x}, s) p(\mathbf{x}, s)\right]+\frac{1}{2} \frac{\partial^{2}}{\partial x^{2}}\left[\sigma_{+}^{2} p(\mathbf{x}, s)\right]$. for $s \geq 0$, with initial condition $p(\mathbf{x}, 0)=\rho^{+}(\mathbf{x})$

Equivalently, the initial distribution of the destinations evolves in time by the Kolmogorov Forward Equation

$\frac{\partial}{\partial s} p(\mathbf{x}, s)=-\frac{\partial}{\partial x}\left[\mathbf{v}^{-}(\mathbf{x}, s) p(\mathbf{x}, s)\right]+\frac{1}{2} \frac{\partial^{2}}{\partial x^{2}}\left[\sigma_{-}^{2} p(\mathbf{x}, s)\right]$. for $s \in\left[t_{i}, t_{f}\right)$, with initial condition $p\left(x, t_{i}\right)=\rho^{-}(x)$.

\section{OPTIMIZATION ON TIME}

Notice that the minimization problem we solved does not really consider the interaction on time because the problem describes the movement of sources and destinations nodes in the space and then we solve the static problem at each time. The problem solved at each time may not be optimal in the whole period of time considered.

Another more realistic problem would be to minimize the quantity of nodes used on the whole network during a fixed period of time $\left[t_{i}, t_{f}\right]$, i.e.,

$$
\begin{gathered}
\operatorname{Min} \int_{t_{i}}^{t_{f}} N(D, t) d t=\int_{t_{i}}^{t_{f}} \int_{D} \eta(\mathbf{x}, t) d \mathbf{x} d t= \\
=\int_{t_{i}}^{t_{f}} \int_{D}|\mathbf{T}(\mathbf{x}, t)|^{2} d \mathbf{x} d t
\end{gathered}
$$

where $N(D, t)$ is the number of active relay nodes in the network $D$ at time $t$.

For the case we have randomness in the system this problem will become

$\operatorname{Min} \int_{t_{i}}^{t_{f}} \mathbb{E}\{N(D, t)\} d t=\int_{t_{i}}^{t_{f}} \int_{D} \mathbb{E}\left\{|\mathbf{T}(\mathbf{x}, t)|^{2}\right\} d \mathbf{x} d t$

subject to (13a), (13b), and (TE) since $D$ is compact. From the work of Santambrogio ([27], page 6) we have the following result: The problem of solving

$$
\operatorname{Min} \int_{D} k(\mathbf{x})|\mathbf{T}(\mathbf{x})| \text { such that } \nabla \cdot \mathbf{T}=\mu-\nu
$$

is equivalent by duality to the problem of finding the shortest path on a curved space (also known as geodesic), i.e.,

$$
\operatorname{Min} \int_{D} d_{k}(x, y) d \gamma \quad \text { such that } \quad \gamma \in \Pi(\mu, \nu)
$$

where $\Pi(\mu, \nu)$ is the set of transport plans between $\mu$ and $\nu$ and the distance $d_{k}(x, y)$ is defined as the minimum between all the trajectories $\omega$ that start at time 0 in $x$ and ends at time 1 in $y$ of the function $L_{k}(\omega)$

$$
d_{k}(x, y)=\operatorname{Min}_{\substack{\alpha=0(0)=x, \omega(1)=y\}}} L_{k}(\omega):=\int_{0}^{1} k(\omega(t))\left|\omega^{\prime}(t)\right| d t
$$

In our case $k(\mathbf{x})=|\mathbf{T}(\mathbf{x})|$ then the function that we want to minimize is

$$
L_{k}(\omega)=\int_{0}^{1}|\mathbf{T}(\omega(t))|\left|\omega^{\prime}(t)\right| d t
$$

Given that $\omega(0)=x$, and $\omega(1)=y$ then by change of variables $L_{k}(\omega)=\int_{x}^{y}|\mathbf{T}(\mathbf{x})| d \mathbf{x}$, and as it is independent of $\omega$ then $d_{k}(x, y)=\int_{x}^{y}|\mathbf{T}(\mathbf{x})| d \mathbf{x}$. 
Example. For the case where we do not have previous knowledge about the velocity drift then we just consider the standard Brownian mobility model given by

$$
d \rho^{+}(t)=\sigma_{+}(\mathbf{x}, t) d W^{+}(t)
$$

and/or

$$
d \rho^{-}(t)=\sigma_{-}(\mathbf{x}, t) d W^{-}(t) .
$$

where $W^{+}(t)$ and $W^{-}(t)$ are two independent Brownian motions with values in $X \times Y$.

Then the previous equations translate into

$$
\begin{aligned}
& \frac{\partial}{\partial s} p(x, s)=+\frac{1}{2} \frac{\partial^{2}}{\partial x^{2}}\left[\sigma_{+}^{2}(x, s) p(x, s)\right] . \\
& \frac{\partial}{\partial s} p(x, s)=+\frac{1}{2} \frac{\partial^{2}}{\partial x^{2}}\left[\sigma_{-}^{2}(x, s) p(x, s)\right] .
\end{aligned}
$$

which have as solution the following equations

$$
\begin{aligned}
& \rho^{+}(x, t)=\frac{1}{\sqrt{2 \pi t \sigma^{+}}} e^{-\frac{x^{2}}{2 t \sigma^{+}}} \\
& \rho^{-}(x, t)=\frac{1}{\sqrt{2 \pi t \sigma^{-}}} e^{-\frac{x^{2}}{2 t \sigma^{-}}}
\end{aligned}
$$

Now we can replace this solution into Step 2 and Step 3.

Remark. Notice that if we suppose that the distribution of the destinations is fixed, as it will be the case for aggregation centers of information, then $\sigma^{-}=0$ and then $\rho^{-}(x, t)=\rho^{-}$for all time $t$.

\section{CONCLUSIONS}

We consider a setting in which a spatially distributed set of mobile sources is creating data for a spatially distributed set of mobile destinations. We make the assumption that the network is massively dense, i.e., there are so many sources, destinations, and nodes, that it is best to describe the network in terms of macroscopic parameters, such as their spatial distribution, rather than in terms of microscopic parameters, such as their individual placements. We focus on a particular physical layer model that is characterized by the following assumptions: i) the wireless nodes must only transport the data from the location of the sources to the location of the destinations, and do not need to sense the data at the sources, or deliver them at the destinations once the data arrive at their physical locations, and ii) the nodes have limited bandwidth available to them, but they use it optimally to locally achieve the network capacity.

In this setting, the optimal distribution of nodes induces a traffic flow that resembles the electric displacement that will be created if we substitute the sources and destinations with positive and negative charges respectively. The analogy between the two settings is very tight, and many features of Electromagnetism have a direct interpretation in wireless sensor networks.

\section{ACKNOWLEDGEMENT}

The authors want to thank Aimé Lachapelle from CEREMADE-Paris Dauphine for helpful discussions. The first author was partially supported by CONICYT Chile and INRIA France. The first, third and fourth authors were partially supported by Alcatel-Lucent within the Alcatel-Lucent Chair in Flexible Radio at Supelec. The mathematical results of Section IV are mainly taken from [25].

\section{REFERENCES}

[1] P. Jacquet, "Geometry of information propagation in massively dense ad hoc networks," in Proc. of MobiHoc '04: Proceedings of the 5th ACM international symposium on Mobile ad hoc networking and computing, pp. 157-162, New York, NY, USA, 2004.

[2] P. Jacquet, "Space-Time Information Propagation in Mobile Adhoc Wireless Networks," IEEE Information Theory Workshop, pp. 260-264, 2004.

[3] M. Kalantari and M. Shayman, "Energy efficient routing in sensor networks," in Proc. of Conference on Information Sciences and Systems. Princeton University, March 2004

[4] M. Kalantari and M. Shayman, "Routing in wireless ad hoc networks by analog to electrostatics theory," in Proc. of IEEE International Communications Conference ICC'04, Paris, France, June 2004.

[5] S. Toumpis and L. Tassiulas, "Packetostatics: Deployment of Massively Dense Sensor Networks as an Electrostatic Problem," IEEE INFOCOM, Vol. 4, pp. 2290-2301, 2005.

[6] S. Toumpis and L. Tassiulas, "Optimal Deployment of Large Wireless Sensor Networks," IEEE Trans. on Information Theory, Vol. 52, No. 7, pp. 2935-2953, Jul. 2006.

[7] S. Toumpis and G. A. Gupta, "Optimal Placement of Nodes in Large Sensor Networks under a General Physical Layer Model" in Proc. of IEEE SECON 2005.

[8] R. Catanuto, G. Morabito, and S. Toumpis, "Optical Routing in Massively Dense Networks: Practical Issues and Dynamic Programming Interpretation." IEEE International Symposium on Wireless Communication Systems, Valencia, Spain, Sep. 2006.

[9] S. Toumpis, "Mother Nature Knows Best: A Survey of Recent Results on Wireless Networks Based on Analogies with Physics," Computer Networks, Vol. 52, No. 2, pp. 360-383, Feb. 2008

[10] E. Altman, A. Silva, P. Bernhard, M. Debbah, "Continuum Equilibria for Routing in Dense Ad-Hoc Networks," 45th Allerton Conference on Communication, Control and Computing, Illinois, USA, Sep. 26-28, 2007.

[11] E. Altman, P. Bernhard, A. Silva, "The Mathematics of Routing in Massively Dense Ad-Hoc Networks," Proc. of AdHoc-NOW Conference, Sophia-Antipolis, France, Sep. 10-13, 2008.

[12] A. Silva, P. Bernhard, E. Altman, "Numerical Solutions of Continuum Equilibria for Routing in Dense Ad-hoc Networks," Proc. of Valuetools, Workshop Inter-Perf, Athens, Greece, Oct. 2024, 2008.

[13] J.G. Wardrop, "Some theoretical aspects of road traffic research," Proceedings of the Institution of Civil Engineers, Part II, I:325378, 1952. 
[14] M. Beckmann, "A continuum model of transportation," Econometrica Vol. 20, pp. 643-660, 1952.

[15] M. Beckmann, C. B. McGuire and C. B. Winsten, Studies in the Economics and Transportation, Yale Univ. Press, 1956.

[16] S. C. Dafermos, "Continuum Modeling of Transportation Networks," Transportation Research Vol. 14B, pp. 295-301, 1980.

[17] P. Daniele and A. Maugeri, "Variational Inequalities and discrete and continuum models of network equilibrium protocols," Mathematical and Computer Modelling 35:689-708, 2002.

[18] H.W. Ho and S.C. Wong, "A Review of the Two-Dimensional Continuum Modeling Approach to Transportation Problems," Journal of Transportation Systems Engineering and Information Technology, Vol.6 No.6 P.53-72, 2006.

[19] G. Idone, "Variational inequalities and applications to a continuum model of transportation network with capacity constraints," Journal of Global Optimization 28:45-53, 2004.

[20] S.C.Wong, Y.C.Du, J.J.Sun and B.P.Y.Loo, "Sensitivity analysis for a continuum traffic equilibrium problem," Ann Reg Sci 40:493$514,, 2006$.

[21] P. Gupta and P. Kumar, "The capacity of wireless networks," IEEE Transactions on Information Theory, Vol. IT-46, No. 2, pp. 388-404, Mar. 2000.

[22] J. Silvester and L. Kleinrock, "On the capacity of multihop slotted ALOHA networks with regular structures," IEEE Trans. Commun., Vol. 31, No. 8, pp. 974-982, Aug. 1983.

[23] M. Franceschetti, O. Dousse, D. Tse, and P. Tiran, "Closing the gap in the capacity of random wireless networks," in Proc. of IEEE ISIT 2004.

[24] R. J. Di Perna and P. L. Lions "Ordinary differential equations, transport theory and Sobolov spaces," Inventiones mathematicae, Vol. 98, pp. 511-547, 1989.

[25] G. Carlier and A. Lachapelle, "A planning problem combining optimal control and optimal transport," available at http://hal.archives-ouvertes.fr/hal-00432785/fr/.

[26] T. Camp, J. Boleng, and B. Davis, "A survey of Mobility Models for Ad-Hoc Networks Research" Wireless Communication and Mobile Computing

[27] F. Santambrogio, "Models and applications of Optimal Transport Theory," Lecture Notes at Summer School 2009, available at http://www-fourier.ujf-grenoble.fr/IMG/pdf/Santambrogio2.pdf 